\title{
Measurement of faecal calprotectin and lactoferrin in inflammatory bowel disease
}

\author{
C A Lamb, ${ }^{1}$ J C Mansfield ${ }^{2}$
}

IInstitute of Cellular Medicine, Newcastle University, Newcastle upon Tyne, UK

${ }^{2}$ Royal Victoria Infirmary, Newcastle upon Tyne Hospitals NHS Foundation Trust, Newcastle upon Tyne, UK

\section{Correspondence to}

Dr Christopher A Lamb, Institute of Cellular Medicine, Newcastle University, The Medical School, Framlington Place, Newcastle upon Tyne NE2 4HH, UK; c.a.lamb@ncl.ac.uk

Accepted 11 October 2010 Published Online First

3 November 2010

\begin{abstract}
Crohn's disease and ulcerative colitis are chronic relapsing gastrointestinal conditions characterised by an influx of inflammatory cells to the affected gut mucosa. The mainstay of diagnosing and re-evaluating these conditions in clinical practice and research is by invasive serological, radiological, endoscopic and histological assessment. In clinical trials, disease activity is often evaluated using a combination of the above tests plus clinical indices such as the Crohn's Disease Activity Index and Ulcerative Colitis Activity Index. These tools rely on subjective assessment of symptoms and so, often, do not correlate with mucosal inflammation or mucosal healing, which may be the preferred therapeutic end point for long-term inflammatory bowel disease (IBD) management.

The faecal biomarkers calprotectin and lactoferrin are neutrophil derived proteins that are stable in faeces and can be detected by quantitative ELISA in small stool samples. Concentrations of both are raised in patients with gastrointestinal mucosal inflammation. They provide a unique, inexpensive, non-invasive method of testing for active inflammatory disease. They can be used to screen for IBD and as a surrogate marker of mucosal healing they are useful in monitoring the response to therapeutic intervention or surgery. They may also predict the clinical course of the disease. This clinical review aims to discuss the current evidence, limitations and potential future uses of these biomarkers in IBD.
\end{abstract}

\section{Introduction}

Crohn's disease and ulcerative colitis (UC) are debilitating chronic relapsing inflammatory conditions for which there is no ideal treatment. There are a growing number of immunological treatments for both diseases, but these carry a risk of side effects and are costly. Consequently, patients must be carefully assessed and counselled about safe and appropriate use of these drugs.

Crohn's disease is often the more clinically challenging of the two conditions to manage. The changing phenotype of the condition often necessitates repeated radiological or endoscopic investigation to evaluate disease activity. These tests are invasive, often uncomfortable, pose risk of short term complications and longterm side effects to the patient, are time consuming and costly.

Historically clinical practice and research trials have relied upon interpretation of symptom activity to determine treatment success in inflammatory bowel disease (IBD). However, over the past 10 years the concept of endoscopic 'mucosal healing' has emerged as a desirable end point of treatment. This suggests that the traditional methods of quantifying clinical activity such as the Crohn's Disease Activity Index (CDAI) and the Ulcerative Colitis Activity Index (UCAI), which predominantly rely on a subjective assessment of symptom severity, may need to be revised or replaced by more objective measurements of mucosal disease activity.

\section{What are calprotectin and lactoferrin?}

Calprotectin is a protein complex of the S-100 family of calcium binding proteins. It is found in high concentrations within neutrophils comprising up to $60 \%$ of protein within the cytosol and is also present in monocytes and macrophages. It is released extracellularly during neutrophil activation or during cell death and also following endothelial adhesion of monocytes. As a result it can be detected and quantified in fluids where inflammation is occurring for example serum, urine, cerebrospinal fluid and faeces. ${ }^{1}$ Calprotectin has antimicrobial and antiproliferative effects that are mediated through zinc chelation, inhibiting metalloproteinases and inducing apoptosis. Interestingly in healthy newborns faecal calprotectin concentrations are significantly elevated in the first month of life. ${ }^{1}$ The rise appears to be most apparent in the first week after birth which may be 
part of the body's physiological defence mechanism against yeasts and fungi, allowing early development of gut homoeostasis.

Lactoferrin is one of the transferrin family of iron binding glycoproteins. It is contained within secondary neutrophil granulocytes but is also expressed in tear fluid, synovial fluid, breast milk and saliva. ${ }^{2}$ It too has an antimicrobial effect, principally through binding to and therefore starving micro-organisms of iron. It is active against bacteria, fungi and viruses. In viral infections it is thought to prevent entry of the virus into the host cell and is active against rotavirus, respiratory syncytial virus, herpes viruses as well as cytomegalovirus and HIV. ${ }^{2}$

During intestinal inflammation both proteins are quickly released into the gut from where they can be quantified within faeces. Both resist proteolysis in the gut lumen and remain remarkably stable within faeces at room temperature for at least 7 days. ${ }^{34}$ This means that samples can be collected by the patient at home and sent by standard mail to the laboratory. When frozen, both proteins remain stable long term allowing delayed analysis. Both can be measured commercially by quantitative ELISA a very small stool sample of $0.05-0.1 \mathrm{~g}$ such that a collection of only a teaspoon sized sample or less is required from the patient. ${ }^{5}$ The normal ranges are well defined as calprotectin $<50 \mu \mathrm{g} / \mathrm{g}$ and lactoferrin $<7.25 \mu \mathrm{g} / \mathrm{g}$ and have been demonstrated as raised in inflammatory, infective and neoplastic enteropathies. ${ }^{6-8}$

The concept of quantifying mucosal inflammation from white cell degradation products in faeces has existed for more than 30 years. Saverymuttu in the 1980s conducted a series of human experiments in patients with IBD infusing intravenous ${ }^{111}$ Indiumlabelled neutophilic granulocytes and measuring excretion through 5 day collection of faeces. The results demonstrated highly significant correlation with CDAI, endoscopic severity, distribution and histological activity. ${ }^{9-11}$ Although this method of investigation has been described as the 'gold standard' method of quantifying mucosal inflammation in IBD, it is cumbersome, time consuming, expensive and exposes patients to ionising radiation. Therefore it is clearly not a test that can be used in routine practice, but these early experiments gave rise to the theory that perhaps there were other faecal biomarkers that could be more easily measured to non-invasively reflect mucosal inflammation. Subsequently in 1999, Roseth and colleagues published the first comparative study of faecal calprotectin measurement with excretion of ${ }^{111}$ Indium-labelled neutrophilic granulocytes in a Norwegian population of patients with Crohn's disease, demonstrating that calprotectin measurement correlated very closely $(\mathrm{p}<0.0001, \mathrm{r}=0.87) .{ }^{12}$ These findings were confirmed by a later United Kingdom (UK) study also demonstrating good correlation between the two biomarkers $(\mathrm{p}<0.0001, \mathrm{r}=0.70){ }^{13}$
The above studies highlighted faecal calprotectin measurement as a practical, safe and non-invasive method of quantifying intestinal inflammation, thus prompting an increasing number of studies in the faecal biomarker field. Subsequently lactoferrin and calprotectin have been identified as the most clinically useful biomarkers. Both correlate strongly with one another, suggesting that measurement of either one is sufficient in clinical practice with little benefit derived from measurement of both biomarkers in individual patients. ${ }^{14} 15$

\section{What are the uses of faecal biomarkers in IBD?}

There are three broad areas within which faecal biomarkers in IBD have been studied: diagnostic exclusion of active IBD, longitudinal monitoring of IBD and predicting the clinical course of IBD.

\section{Diagnostic exclusion of active IBD}

Abdominal pain and diarrhoea are common presenting symptoms to gastroenterology clinics. Without 'red flag' or specific localising symptoms the diagnostic spectrum is wide, from IBD and colonic cancer to functional diarrhoea and irritable bowel syndrome (IBS). Patients are often subjected to colonoscopy or radiological imaging. Consequently, faecal calprotectin and lactoferrin have been studied with an aim of separating those patients with inflammatory organic pathology from those with non-inflammatory functional pathology, thereby potentially avoiding the need for more invasive tests.

Faecal biomarkers are a measure of local gut inflammation rather than systemic inflammation which is not always apparent in IBD. It is not surprising therefore, that calprotectin and lactoferrin have been demonstrated as more effective in identifying disease activity than serum measurements of C-reactive peptide, leucocyte count or erythrocyte sedimentation rate. ${ }^{14}{ }^{16-18}$

It has been demonstrated that both calprotectin and lactoferrin levels are significantly higher in patients with active IBD than in healthy controls and those with IBS. In this setting the overall sensitivity and specificity for calprotectin may be as high as $78-100 \%$ and 76-100\%, respectively, with a similar sensitivity and specificity for lactoferrin of $67-91 \%$ and $90-100 \%$, respectively. ${ }^{67131619-29} \mathrm{~A}$ meta-analysis of facecal calprotectin studies revealed that at a cut off point of $50 \mu \mathrm{g} / \mathrm{g}$ (ie, above the normal range) calprotectin has a sensitivity and specificity of $89 \%$ and $81 \%$ in discriminating IBD from non-IBD diagnoses. ${ }^{30}$ The same paper shows that at a threshold of $100 \mu \mathrm{g} / \mathrm{g}$ (twice upper limit of normal) the sensitivity and specificity improve to $98 \%$ and 91\%, respectively. A normal biomarker result in a patient with active symptoms is therefore very reassuring and may be enough, in the correct context where the clinical risk of malignancy is low, to avoid further colonic investigation such as radiology or colonoscopy and allow a positive diagnosis of functional diarrhoea or IBS at first clinic visit. 
A recent meta-analysis from the Netherlands has concluded an impressive potential reduction in the number of adults requiring colonoscopy by $67 \%$ and children by $35 \%$ through incorporating faecal calprotectin as a screening test for IBD. ${ }^{29}$ However the authors do caution that false negative results may lead to delayed diagnosis in $6 \%$ of adults and $8 \%$ of children.

Faecal biomarkers also have a role in assessing the small intestine, often beyond the reach of conventional colonoscopy as demonstrated by a UK study that compared calprotectin to small bowel barium follow through. ${ }^{31}$ Nine of 25 patients with active Crohn's disease had abnormal radiology. In all cases calprotectin was raised to $>60 \mu \mathrm{g} / \mathrm{g}$ and the authors found that a calprotectin $<60 \mu \mathrm{g} / \mathrm{g}$ had a $100 \%$ negative predictive value for an abnormal follow through result.

Faecal lactoferrin has also been compared with faecal occult blood (FOB) testing for its predictive capabilities in colorectal disease. A study of 351 patients attending for colonoscopy who gave a stool sample the preceding day found $\mathrm{FOB}$ and lactoferrin to have equal specificity $(88.7 \%)$ in predicting organic pathology including IBD, colorectal cancer and polyps. ${ }^{32}$

\section{Longitudinal monitoring of IBD}

For many years treatment success and trial efficacy in IBD has been defined by symptom resolution. When the CDAI was created almost 35 years ago, it was developed as a method of standardising entry into clinical trials but not as a method of individually monitoring patients. ${ }^{33}$ Although CDAI and UCAI may give an indirect suggestion of disease activity, they are not direct measures of mucosal inflammation and may be raised by non-inflammatory symptoms. Faecal biomarkers may challenge the supremacy of the CDAI and UCAI by providing a real time indication of disease activity that can be measured longitudinally and that correlates well with clinical, endoscopic and histological activity in IBD.

It has previously been shown that normalisation of calprotectin is a surrogate marker of endoscopic mucosal healing in patients being treated for active IBD. ${ }^{5}$ Mucosal healing may be the optimal outcome of therapy in IBD, as it is associated with more sustained clinical remission and a reduced need for surgery. ${ }^{34} \mathrm{~A}$ raised or normal biomarker result in a patient with IBD can therefore be a very useful decision making aid when investigating changing symptoms that may be due to an inflammatory flare of the disease, co-existing IBS, non-inflammatory complications (eg, adhesions), or post operative changes such as bile acid malabsorption or short bowel syndrome. ${ }^{14}$

A recent study has looked at whether calprotectin can give insight into the symptoms of patients previously labelled as having concomitant IBS and IBD. The study found that $59.7 \%$ of patients with Crohn's disease and $38.6 \%$ of patients with UC in clinical remission fulfilled the Rome II criteria for IBS. ${ }^{35}$ However, they found that in these groups, calprotectin levels were significantly elevated above normal and were higher than in those patients without IBS symptoms. This suggests that some patients may be mislabelled as having concomitant IBS when in fact they have uncontrolled IBD driving their symptoms. Consequently, calprotectin may be able to help identify those patients who may benefit from disease-modifying treatment rather than symptomatic treatment.

Many studies have shown both biomarkers to correlate strongly with invasive methods of determining extent and severity of mucosal inflammation such as 99-technetium white cell scanning and colonoscopy in both adults and children. ${ }^{36}{ }^{37}$ Equally, overall the biomarkers have been found to correlate well with clinical disease activity. ${ }^{16} 171921$ However there have been some exceptions demonstrating poor clinical correlation between CDAI and faecal biomarkers, but good correlation between endoscopic activity and faecal biomarkers. ${ }^{38}$ Rather than this representing a flaw in the use of biomarkers, it further highlights the problem that clinical indices of disease activity can underestimate the level of mucosal inflammation.

Furthermore it has been shown that calprotectin correlates better with histological disease activity than macroscopic inflammation judged by clinicians at endoscopy. Again this suggests that biomarkers can provide accurate non-invasive information regarding mucosal disease activity, without necessarily the need for colonoscopy. ${ }^{36}$

There are limited but very encouraging studies regarding faecal biomarkers to assess the response to infliximab treatment in Crohn's disease. Faecal lactoferrin has been measured in a paediatric population of five patients with severe Crohn's disease as determined by the paediatric CDAI receiving infliximab induction. ${ }^{39}$ Within 7-10 days the concentration of faecal lactoferrin reduced by $93.43 \pm 4.49 \%$ towards baseline, probably reflecting mucosal healing although endoscopy was not performed to correlate this. A further study endoscopically assessing response to treatment found that in those who achieved endoscopic remission after infusion, faecal biomarkers fell from a pre-infusion mean of $1891 \mu \mathrm{g} / \mathrm{g}$ calprotectin (range 813-2434) and $92.4 \mu \mathrm{g} / \mathrm{g}$ lactoferrin (range 35.5-235.6), to a normal post-infusion mean of $27 \mu \mathrm{g} / \mathrm{g}$ calprotectin (range 13-130) and $1.9 \mu \mathrm{g} / \mathrm{g}$ lactoferrin (range 0.0-2.1), therefore providing a surrogate marker of response and also mucosal healing. ${ }^{40}$

Faecal biomarkers may also be of clinical use to monitor Crohn's disease following intestinal resection. In this setting inflammatory relapse is common, but can be difficult to identify clinically as symptoms may be due to IBS, bile salt malabsorption or altered gut anatomy, often necessitating radiological or endoscopic investigation. A UK study has shown in the normal postoperative period both lactoferrin and calprotectin normalise within 2 months. Therefore a 
single biomarker measurement in patients with symptoms after this time point may aid decision making about further investigation for surgical complications or disease recurrence if the biomarker is positive, or a trial of conservative treatment such as loperamide or cholestyramine if the biomarker is within the normal range. ${ }^{14}$

Further support for the use of biomarkers postoperatively can be derived from an Italian paper of biomarker correlation to disease activity. ${ }^{23}$ In this study 12 patients had undergone surgery for Crohn's disease and eight had disease recurrence involving the anastomosis, all of whom had a positive calprotectin. Of the four with no recurrence at the anastomosis none had an elevated calprotectin. Similarly a positive lactoferrin in a symptomatic patient following ileal pouch-anal anastomosis surgery has been demonstrated to give a sensitivity of $100 \%$ and specificity of $85 \%$ in distinguishing between active Crohn's disease, pouchitis and cuffitis, versus irritable pouch syndrome, in which inflammation is not driving symptoms. ${ }^{8}$

\section{Predicting the clinical course of IBD}

An important question is whether single or sequential measurement of faecal biomarkers can predict the future course of IBD? If so, their use in clinical practice is potentially vast, allowing early counselling regarding the need for institution of immunosuppressive or biological drugs, or surgery. Equally biomarker measurement may allow discontinuation of unnecessary drugs in those at high likelihood of staying in remission long term.

A UK study monitored 43 patients with Crohn's disease and 37 patients with UC whom had been in clinical remission for between 1 and 4 months. ${ }^{41}$ An index calprotectin $>50 \mathrm{mg} / \mathrm{l}$ (an older unit measurement of calprotectin that equates to $>250 \mu \mathrm{g} / \mathrm{g}$ ) predicted clinical relapse over the next 12 months with a sensitivity of $90 \%$ and a specificity of $83 \%$.

Similarly another study followed up 38 patients with Crohn's disease and 41 patients with UC in clinical remission, again for 1 year. ${ }^{42}$ An index calprotectin of $>150 \mu \mathrm{g} / \mathrm{g}$ was a poor indicator of relapse according to CDAI in Crohn's disease, conferring only a twofold risk of relapse, with a sensitivity of $87 \%$ but a specificity of only $47 \%$. However, in UC the same calprotectin threshold gave rise to an impressive 14-fold risk of relapse, with a sensitivity of $89 \%$ and a specificity of $82 \%$.

\section{What cautions and limitations are there of using faecal biomarkers?}

It is important to remember that despite all of this promising data, faecal biomarker quantification as a screening test for IBD cannot fully replace conventional endoscopy and radiology. IBD remains a histological diagnosis requiring intestinal biopsies, so a positive calprotectin or lactoferrin will require further tests not only to confirm or refute a diagnosis of IBD, but also to exclude other causes of raised biomarkers including malignancy, polyps, viral or bacterial gastroenteritis, NSAID enteropathy, untreated coeliac disease or gastro-oesophageal reflux disease. ${ }^{27}$ Therefore the use of faecal biomarkers should be restricted to situations where the result along with clinical judgement informs a decision about treatment, allows avoidance of invasive tests, or where invasive tests have not provided a satisfactory conclusion-for example, a normal colonoscopy but clinical suspicion of small bowel inflammation.

It is also necessary to appreciate that inflammation is only one component of IBD and it is only with regard to this that faecal biomarkers are of clinical use. Symptomatic patients with IBD who have normal biomarkers should not simply be labelled as having IBS without further consideration. Patients may have symptoms due to non-inflammatory mechanical disease such as bile salt malabsorption, fibrotic strictures or adhesions and these are likely to be best treated by identifying the correct problem and giving specific treatment.

Furthermore, symptoms remain the most powerful mediators of patient behaviour and decision making. As such the greatest challenge to incorporating faecal biomarkers into management pathways may be when there is disparity between how a patient feels and what faecal biomarkers inform us regarding their current inflammatory activity. For instance, it may be very difficult trying to encourage a patient with no symptoms and a positive faecal biomarker that they should begin powerful immunosuppressant treatment that poses risk of side effect.

\section{Conclusions and the future for faecal biomarkers in IBD}

The outlook for faecal calprotectin and lactoferrin in clinical practice appears promising.

Potentially in the future faecal biomarker measurement will be standard prior to first gastrointestinal outpatient clinic visit for those with abdominal pain or diarrhoea. This may allow faster triage and assessment of patients and perhaps the avoidance of unnecessary investigation in those with functional diarrhoea or IBS.

As surrogate markers of mucosal healing in IBD, calprotectin and lactoferrin may allow objective mapping of an individual patient response to treatment and quantify the likelihood of future relapse. If these tests can be used to demonstrate an adequate response to biological therapy, they may aid decisions regarding dose escalation, shortening of dosage interval, switching to an alternative agent or ultimately withdrawal of unnecessary immunosuppression for those in sustained deep remission. Equally future trials may tell us whether consistent suppression or elevation of calprotectin and lactoferrin convey prognostic significance in terms of hospitalisation, the need for surgery and an effect on quality of life. 


\section{Key points}

- Quantification of the faecal biomarkers calprotectin and lactoferrin can provide a non-invasive measure of intestinal inflammation.

- The clinical use of faecal biomarkers in inflammatory bowel disease includes screening for active mucosal inflammation, longitudinal disease monitoring and predicting the risk of relapse.

- In the correct clinical context, faecal biomarkers may avoid the need for invasive radiological or endoscopic investigation and inform treatment decisions.

In summary, faecal biomarkers will never fully replace colonoscopy and radiology which are necessary to obtain tissue samples and investigate the complications of IBD. However, in a society where patient satisfaction, risk minimisation, cost reduction and hospitalisation avoidance are a priority, these noninvasive, inexpensive, reproducible and clinically significant measurements are likely to have a greater role in our future diagnostic and therapeutic pathways.

Competing interests CL has previously received an educational grant from TechLab, Inc who manufacture the IBD-SCAN ELISA testing kit for faecal lactoferrin. TechLab, Inc have previously supplied JCM with IBD-SCAN ELISA testing kits for research into postoperative Crohn's disease.

\section{Provenance and peer review Commissioned;} externally peer reviewed.

\section{References}

1. Baldassarre ME, Altomare MA, Fanelli M, et al. Does calprotectin represent a regulatory factor in host defense or a drug target in inflammatory disease? Endocr Metab Immune Disord Drug Targets 2007;7:1-5.

2. Levy O. Antimicrobial proteins and peptides of blood: templates for novel antimicrobial agents. Blood 2000;96:2664-72.

3. Røseth AG, Fagerhol MK, Aadland E, et al. Assessment of the neutrophil dominating protein calprotectin in feces. A methodologic study. Scand J Gastroenterol 1992;27:793-8.

4. Guerrant RL, Araujo V, Soares E, et al. Measurement of fecal lactoferrin as a marker of fecal leukocytes. J Clin Microbiol 1992;30:1238-42.

5. Røseth AG, Aadland E, Grzyb K. Normalization of faecal calprotectin: a predictor of mucosal healing in patients with inflammatory bowel disease. Scand J Gastroenterol 2004;39:1017-20.

6. Fagerberg UL, Lööf L, Myrdal U, et al. Colorectal inflammation is well predicted by fecal calprotectin in children with gastrointestinal symptoms. J Pediatr Gastroenterol Nutr 2005;40:450-5.

7. Kane SV, Sandborn WJ, Rufo PA, et al. Fecal lactoferrin is a sensitive and specific marker in identifying intestinal inflammation. Am J Gastroenterol 2003;98:1309-14.

8. Parsi MA, Shen B, Achkar JP, et al. Fecal lactoferrin for diagnosis of symptomatic patients with ileal pouch-anal anastomosis. Gastroenterology 2004;126:1280-6.

9. Saverymuttu SH, Camilleri M, Rees H, et al. Indium 111granulocyte scanning in the assessment of disease extent and disease activity in inflammatory bowel disease. A comparison with colonoscopy, histology and fecal indium 111-granulocyte excretion. Gastroenterology 1986;90(5 Pt 1):1121-8.

10. Saverymuttu SH, Peters AM, Hodgson HJ, et al. Assessment of disease activity in ulcerative colitis using indium-111-labelled leukocyte faecal excretion. Scand J Gastroenterol 1983;18:907-12.

11. Saverymuttu SH, Peters AM, Lavender JP, et al. Quantitative fecal indium 111-labeled leukocyte excretion in the assessment of disease in Crohn's disease. Gastroenterology 1983;85:1333-9.

12. Røseth AG, Schmidt PN, Fagerhol MK. Correlation between faecal excretion of indium-111-labelled granulocytes and calprotectin, a granulocyte marker protein, in patients with inflammatory bowel disease. Scand J Gastroenterol 1999;34:50-4.

13. Tibble J, Teahon K, Thjodleifsson B, et al. A simple method for assessing intestinal inflammation in Crohn's disease. Gut 2000;47:506-13.

14. Lamb CA, Mohiuddin MK, Gicquel J, et al. Faecal calprotectin or lactoferrin can identify postoperative recurrence in Crohn's disease. Br J Surg 2009;96:663-74.

15. Scarpa M, D'Incà R, Basso D, et al. Fecal lactoferrin and calprotectin after ileocolonic resection for Crohn's disease. Dis Colon Rectum 2007;50:861-9.

16. Schoepfer AM, Trummler M, Seeholzer P, et al. Discriminating IBD from IBS: comparison of the test performance of fecal markers, blood leukocytes, CRP and IBD antibodies. Inflamm Bowel Dis 2008;14:32-9.

17. Sipponen T, Savilahti E, Kolho KL, et al. Crohn's disease activity assessed by fecal calprotectin and lactoferrin: correlation with Crohn's disease activity index and endoscopic findings. Inflamm Bowel Dis 2008;14:40-6.

18. Tibble JA, Sigthorsson G, Foster R, et al. Use of surrogate markers of inflammation and Rome criteria to distinguish organic from nonorganic intestinal disease. Gastroenterology 2002;123:450-60.

19. Schoepfer AM, Trummler M, Seeholzer P, et al. Accuracy of four fecal assays in the diagnosis of colitis. Dis Colon Rectum 2007;50:1697-706.

20. Schröder O, Naumann M, Shastri Y, et al. Prospective evaluation of faecal neutrophil-derived proteins in identifying intestinal inflammation: combination of parameters does not improve diagnostic accuracy of calprotectin. Aliment Pharmacol Ther 2007;26:1035-42.

21. Walker TR, Land ML, Kartashov A, et al. Fecal lactoferrin is a sensitive and specific marker of disease activity in children and young adults with inflammatory bowel disease. J Pediatr Gastroenterol Nutr 2007;44:414-22.

22. Otten CM, Kok L, Witteman BJ, et al. Diagnostic performance of rapid tests for detection of fecal calprotectin and lactoferrin and their ability to discriminate inflammatory from irritable bowel syndrome. Clin Chem Lab Med 2008;46:1275-80.

23. Costa F, Mumolo MG, Bellini M, et al. Role of faecal calprotectin as non-invasive marker of intestinal inflammation. Dig Liver Dis 2003;35:642-7.

24. D'Incà R, Dal Pont E, Di Leo V, et al. Calprotectin and lactoferrin in the assessment of intestinal inflammation and organic disease. Int J Colorectal Dis 2007;22:429-37.

25. Thjodleifsson B, Sigthorsson G, Cariglia N, et al. Subclinical intestinal inflammation: an inherited abnormality in Crohn's disease relatives? Gastroenterology 2003;124:1728-37.

26. Carroccio A, Iacono G, Cottone M, et al. Diagnostic accuracy of fecal calprotectin assay in distinguishing organic causes of chronic diarrhea from irritable bowel syndrome: a prospective study in adults and children. Clin Chem 2003;49(6 Pt1):861-7. 
27. Berni Canani R, Rapacciuolo L, Romano MT, et al. Diagnostic value of faecal calprotectin in paediatric gastroenterology clinical practice. Dig Liver Dis 2004;36:467-70.

28. Sidhu R, Wilson P, Wright A, et al. Faecal lactoferrin-a novel test to differentiate between the irritable and inflamed bowel? Aliment Pharmacol Ther 2010;31:1365-70.

29. van Rheenen PF, Van de Vijver E, Fidler V. Faecal calprotectin for screening of patients with suspected inflammatory bowel disease: diagnostic meta-analysis. BMJ 2010;341:c3369.

30. von Roon AC, Karamountzos L, Purkayastha S, et al. Diagnostic precision of fecal calprotectin for inflammatory bowel disease and colorectal malignancy. Am J Gastroenterol 2007;102:803-13.

31. Dolwani S, Metzner M, Wassell JJ, et al. Diagnostic accuracy of faecal calprotectin estimation in prediction of abnormal small bowel radiology. Aliment Pharmacol Ther 2004;20:615-21.

32. Saitoh O, Kojima K, Kayazawa M, et al. Comparison of tests for fecal lactoferrin and fecal occult blood for colorectal diseases: a prospective pilot study. Intern Med 2000;39: 778-82.

33. Best WR, Becktel JM, Singleton JW, et al. Development of a Crohn's disease activity index. National Cooperative Crohn's Disease Study. Gastroenterology 1976;70:439-44.

34. Schnitzler F, Fidder H, Ferrante M, et al. Mucosal healing predicts long-term outcome of maintenance therapy with infliximab in Crohn's disease. Inflamm Bowel Dis 2009;15:1295-301.
35. Keohane J, O'Mahony C, O'Mahony L, et al. Irritable bowel syndrome-type symptoms in patients with inflammatory bowel disease: a real association or reflection of occult inflammation? Am J Gastroenterol 2010;105:1788, 89-94; quiz 95.

36. Bunn SK, Bisset WM, Main MJ, et al. Fecal calprotectin: validation as a noninvasive measure of bowel inflammation in childhood inflammatory bowel disease. J Pediatr Gastroenterol Nutr 2001;33:14-22.

37. Gaya DR, Lyon TD, Duncan A, et al. Faecal calprotectin in the assessment of Crohn's disease activity. QJM 2005;98:435-41.

38. Jones J, Loftus EV Jr, Panaccione R, et al. Relationships between disease activity and serum and fecal biomarkers in patients with Crohn's disease. Clin Gastroenterol Hepatol 2008;6:1218-24.

39. Buderus S, Boone J, Lyerly D, et al. Fecal lactoferrin: a new parameter to monitor infliximab therapy. Dig Dis Sci 2004;49:1036-9.

40. Sipponen T, Savilahti E, Kärkkäinen P, et al. Fecal calprotectin, lactoferrin and endoscopic disease activity in monitoring antiTNF-alpha therapy for Crohn's disease. Inflamm Bowel Dis 2008;14:1392-8.

41. Tibble JA, Sigthorsson G, Bridger S, et al. Surrogate markers of intestinal inflammation are predictive of relapse in patients with inflammatory bowel disease. Gastroenterology 2000;119:15-22.

42. Costa F, Mumolo MG, Ceccarelli L, et al. Calprotectin is a stronger predictive marker of relapse in ulcerative colitis than in Crohn's disease. Gut 2005;54:364-8. 